\section{Kidney \\ Blood Pressure Research}

Kidney Blood Press Res 2016;41:119-128

DOI: 10.1159/000443413

Published online: February 20, 2016

Accepted: January 13, 2016

\title{
Mesenchymal Stromal Cell-Derived Extracellular Vesicles Protect Against Acute Kidney Injury Through Anti-Oxidation by Enhancing Nrf2/ARE Activation in Rats
}

\author{
Guangyuan Zhang $^{\mathrm{a}}$ Xiangyu Zou ${ }^{\mathrm{b}} \quad$ Yeqing Huang $^{\mathrm{a}}$ Feng Wang $^{\mathrm{c}}$ Shuai Miao \\ Guohua Liub Ming Chen ${ }^{\mathrm{a}}$ Yingjian Zhu ${ }^{\mathrm{d}}$ \\ aDepartment of Urology, Zhongda Hospital, School of Medicine, Southeast University, Nanjing, \\ 'Department of Urology, Shanghai First People's Hospital, School of Medicine, 'Department of \\ Nephrology and Rheumatology, Affiliated Sixth People's Hospital, 'Department of Urology, Xinhua \\ Hospital, School of Medicine, Shanghai Jiao Tong University, Shanghai, China
}

\section{Key Words}

Mesenchymal stromal cells $•$ Extracellular vesicles $•$ Acute kidney injury $\bullet$ Ischemia-reperfusion injury • Nrf2 • HO-1

\begin{abstract}
Background/Aims: Anti-oxidation is an effective strategy for curing acute kidney injury (AKI). Herein, we suggest that extracellular vesicles (EVs) derived from mesenchymal stromal cells (MSCs) might play an anti-oxidative role by enhancing Nrf2/ARE activation in AKI. Methods: EVs isolated from the conditioned medium of human Wharton's Jelly mesenchymal stromal cells and human foreskin fibroblast were intravenously injected in rats immediately after 45 min of unilateral kidney ischemia. Animals were sacrificed $24 \mathrm{~h}$ after injury. Results: Results showed that renal tubular injury was alleviated and renal function was improved by MSC-EVs. Cell apoptosis and SNGAL levels, which reflect kidney cell injury, were reduced. Moreover, MSC-EVs decreased oxidative stress in injured kidney tissues and NRK-52E cells under hypoxia injury. Nrf2/antioxidant response element (ARE) enhancement and HO-1 up-regulation were further observed after MSC-EV treatment both in vivo and in vitro. Conclusions: MSC-EVs may protect against AKI possibly through anti-oxidation by enhancing Nrf2/ARE activation.
\end{abstract}

G. Zhang, X. Zou and Y. Huang contributed equally to this study and therefore share first authorship.

Dr. Yingjian $\mathrm{Zhu}^{1}$, and

Dr. Ming Chen ${ }^{2}$ 


\section{Kidney Blood Pressure Research}

Kidney Blood Press Res 2016;41:119-128

\begin{tabular}{l|l}
\hline DOI: 10.1159/000443413 & (C) 2016 The Author(s). Published by S. Karger AG, Base
\end{tabular}

Published online: February 20, 2016

www.karger.com/kb

\section{Introduction}

Acute kidney injury (AKI), a severe syndrome with adverse outcomes [1], is commonly seen in clinical practice, especially after renal transplantation [2], partial nephrectomy [3] and cardiac surgery [4]. AKI mortality rates are alarmingly high and range from $24 \%$ to $62 \%$ [5]. Therefore, several therapeutic strategies for AKI, such as mesenchymal stromal cell (MSC) transplantation, have been developed. However, MSC transplantation presents some limitations, such as immune-mediated rejection and malignancy transformation [6]. Thus, a number of researchers have suggested 'cell-free therapy', which uses extra-cellular vesicles (EVs) of MSCs for injury therapy [6, 7].

EVs, also called micro-vesicles or micro-particles, are membranous structures released by various cells. EVs can deliver bioactive molecular contents such as proteins, DNA, mRNAs, miRNA and other nuclear materials, and are described as novel pathways of inter-cellular communications $[8,9]$. EVs from MSCs can also play a protective role in kidney injury [1012]; however, the mechanism of this action remains unclear.

Oxidative stress caused by increased production of reactive oxygen species (ROS) [13] leads to necrosis, apoptosis, inflammation and other disorders in AKI. The endogenous protective system could be activated when oxidative stress is present, which means the antioxidant is produced to catalyse or neutralise ROS and subsequently diminish tissue injury [14]. Nuclear factor E2-related factor 2 (Nrf2), a transcription factor identified in 1994 [15], is referred to as the 'master regulator' of antioxidant responses. Activated Nrf2 enters the nucleus and binds to antioxidant response elements (AREs), thereby modulating the expression of large numbers of antioxidant $[16,17]$.

MSCs protect kidneys against ischemia-reperfusion injury (IRI) by suppressing oxidative stress [18]; however, the relevant mechanism remains unclear. One study has demonstrated that MSCs can restore $\mathrm{CCl}_{4}$-induced liver injury by enhancing Nrf2 activation [19]. Thus, we deduced that MSCs derived from EVs may also play an anti-oxidative role in AKI recovery by enhancing the Nrf2/ARE pathway.

In this study, we investigate the anti-oxidative role of human Wharton's Jelly mesenchymal stromal cell (hWJMSC)-secreted EVs in AKI recovery and test our hypothesis that EVs can enhance the Nrf2/ARE pathway.

\section{Materials and Methods}

\section{Ethics statement}

In this study, all research involving human participants was approved by the institutional review board of the Chinese Academy of Medical Science and Southeast University. The subjects provided their written informed consent to participate in the research and allowed us to publish case details. This study was conducted in strict accordance with the recommendations from the Guide for the Care and Use of Laboratory Animals of Southeast University. The protocol was approved by the Committee on the Ethics of Animal Experiments of Southeast University. All surgeries were performed under sodium pentobarbital anaesthesia, and all efforts were made to minimise suffering.

\section{Cell culture}

Healthy human umbilical cords were collected and stored in cold Hank's balanced salt solution (Sigma-Aldrich, USA); cellular isolation was initiated within $4 \mathrm{~h}$. hWJMSC isolation and identification were performed as previously described [20]. After isolation, the MSCs were cultured in low-glucose Dulbecco's modified eagle's medium (DMEM, Gibco, USA) containing $10 \%$ foetal bovine serum (FBS, Gibco) at $37^{\circ} \mathrm{C}$ in a humidified atmosphere with $5 \% \mathrm{CO}_{2}$. Only those cells from the third to sixth passages were used for the experiments. NRK-52E cells were obtained from a commercial source (Shanghai Institutes for Biological Sciences, Shanghai, China) and cultured in low-glucose DMEM containing 5\% FBS and $1.5 \mathrm{~g} / \mathrm{L}$ sodium bicarbonate. Human foreskin fibroblasts (HFFs) were also obtained from a commercial source (Shanghai Institutes for Biological Sciences) and cultured in RPMI-1640 (Gibco) supplemented with 10\% FBS. 


\section{Kidney Blood Pressure Research}

EVs extraction and characterisation

EVs were obtained from hWJMSC and HFF supernatants as previously described [20-22]. In brief, cells were cultured in FBS-deprived medium and supplemented with $0.5 \%$ bovine serum albumin (BSA, SigmaAldrich, USA) overnight. The conditioned medium (CM) was collected on day 2. After centrifugation at 2,000 $\times g$ for 20 min to remove the debris, the CM was ultra-centrifuged at 100,000 $\times g$ in an SW41 swing rotor (Beckman Coulter Optima L-80K Ultracentrifuge; Beckman Coulter, Fullerton, CA, USA) for $1 \mathrm{~h}$ at $4{ }^{\circ} \mathrm{C}$. EVs were washed once with serum-free M199 containing 25 mM HEPES (pH 7.4) and submitted to a second cycle of ultracentrifugation under the same conditions. EVs pellets were suspended in serum free M199 and protein content quantified using the Bradford assay. A total of $100 \mu \mathrm{g}$ of EVs could be achieved from about $5 \times 10^{5}$ cells after serum-free overnight.

EVs characterization was performed as previously described [22]. EVs were morphologically analysed using transmission electron microscopy, and flow cytometric assay was used to determine the EV surface marker.

Rat AKI model induced by IRI

Eight-week old male rats (180-200 g) were used in the experiments and housed under constant temperature and humidity, with a $12 \mathrm{~h}: 12 \mathrm{~h}$ light/dark cycle. The AKI model was set up as previously described [23]. In brief, the rats were subjected to right nephrectomy after induction of isofluorane anaesthesia. Left renal ischemia was induced by non-traumatic vascular clamps over the renal artery for $45 \mathrm{~min}$. Reperfusion was then established. The same procedure was performed on sham-treated animals, except that the renal vessels were not clamped. A total of $100 \mu \mathrm{g}$ of EVs (from MSC or HFF) in $1 \mathrm{~mL}$ of vehicle (M199) or $1 \mathrm{~mL}$ of vehicle only was administered through the caudal vein immediately after reperfusion ( $\mathrm{n}=6$ for each group). Rats were sacrificed $24 \mathrm{~h}$ later, and blood and left kidneys were collected for examination.

Hypoxia re-oxygenation injury for $N R K-52 E$

NRK-52E cells were injured through $6 \mathrm{~h}$ of hypoxia $\left(5 \% \mathrm{O}_{2}\right)$ and incubated by exposure to EVs (MSC or $\mathrm{HFF}$ ) or the vehicle only (M199) for $24 \mathrm{~h}$ at $37^{\circ} \mathrm{C}$ in a humidified atmosphere with $5 \% \mathrm{CO}_{2}$. Uninjured NRK$52 \mathrm{E}$ cells were used as controls.

Renal function

Serum creatinine was measured using colorimetric microplate assay based on the Jaffe reaction (BioAssay Systems, Hayward, CA, USA). A colorimetric assay kit was used to quantify the levels of blood urea nitrogen (BUN) in accordance with the supplier's protocol (BioAssay Systems).

\section{Histological examinations}

Part of the left kidney was fixed in 10\% formalin, dehydrated in ethanol and embedded in paraffin. Kidney tissue blocks were cut into $4 \mu \mathrm{m}$ sections and subjected to periodic acid Schiff (PAS) staining. The sections were viewed under light microscopy. Histological scoring was assessed using the following criteria: 0 , none; $1,0-10 \% ; 2,11 \%-25 \% ; 3,26 \%-45 \% ; 4,46 \%-75 \%$; and $5,76 \%-100 \%$, as previously described [24].

Renal cell apoptosis was assessed by TUNEL staining (Roche Diagnostics, Mannheim, Germany) for different groups, as previously described [25].

\section{Lipid peroxide examination}

Malondialdehyde (MDA), a product of reduction during lipid peroxidation [26], was detected from the supernatant of the renal cortical homogenate using a commercial assay kit (Nanjing Jiancheng Bioengineering Institute, Jiangsu, China) according to the manufacturer's instruction.

\section{ELISA assay}

Serum neutrophil gelatinase-associated lipocalin (sNAGL) was measured using commercial ELISA kits (R\&D, USA). 8-OHdg concentrations were also assessed using commercial ELISA kits (Japan Institute for the Control of Aging, Shizuoka, Japan). 


\section{Kidney Blood Pressure Research}

Superoxide dismutase (SOD) activity

A commercial assay kit (Nanjing Jiancheng Bioengineering Institute) was utilised to detect SOD activity. The assay was performed according to the manufacturer's instructions.

ROS assay for NRK-52E

ROS level assay was conducted as previously described [27-29]. After EV or vehicle exposure for 24h (meanwhile, the nuclei were stained with 4',6-diamidino-2-phenylindole (DAPI, $0.5 \mu \mathrm{g} / \mathrm{ml}$, Sigma-Aldrich)

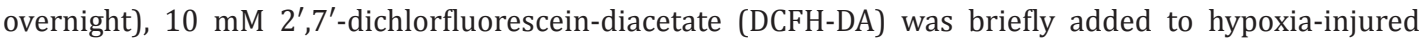
NRK-52E cells. Cells without injury were also assayed as controls. The cells were incubated for $20 \mathrm{~min}$ at $37^{\circ} \mathrm{C}$ and washed in serum-free medium thrice to remove extracellular DCFH-DA. Intracellular ROS levels in the NRK-52E cells were detected by determining the fluorescence intensity of $2^{\prime}, 7^{\prime}$-dichlorofluorescein under a fluorescence microscope. The Fluorescence intensity was also detected using a fluorescence spectrophotometer at excitation and emission wavelengths of 488 and $525 \mathrm{~nm}$, respectively.

Western blot analysis of Nrf2 and HO-1

Cytoplasmic and nuclear proteins were extracted from renal tissues using a nuclear extract kit (Active Motif, Tokyo, Japan) according to the manufacturer's instructions. Target proteins were measured through Western blot analysis using rabbit antibodies against rat Nrf2 or HO-1 (Santa Cruz, USA). Antibodies to histone H3 (Cell Signalling Technology, USA) or $\beta$-actin (Santa Cruz) were used as internal controls for the nuclear and cytosolic target proteins, respectively.

ARE reporter assay

ARE reporter assay was used to confirm the transcriptional activity of Nrf2 on NRK-52E using a Signal ${ }^{\mathrm{TM}}$ ARE Reporter Assay Kit (SABiosciences). The assay was performed according to the manufacturer's instructions.

\section{Quantitative real-time PCR}

The total RNA from NRK-52E cells or kidney tissues was extracted using TRizol reagent (Invitrogen). mRNA expression levels were quantified in total RNA using real-time PCR with Taqman chemistry (Applied Biosystems, USA), as previously described [23]. GAPDH was used as the internal normaliser for mRNAs. The primers of rat HO- 1 and GAPDH were 5 '-accccaccaagttcaaacag- 3 ' (forward), 5'-gattctggcggaaggacgag- $3^{\prime}$ (reverse) and $5^{\prime}$ tgtccagcctcgtctcatag- $3^{\prime}$ (forward), $5^{\prime}$-ctgagatgggtgccgttcaag- $3^{\prime}$ (reverse), respectively. The one-step qPCR method was used, and each experiment was performed in triplicate in clear 384-well plates at $48^{\circ} \mathrm{C}$ for $30 \mathrm{~min}, 95^{\circ} \mathrm{C}$ for $10 \mathrm{~min}, 95^{\circ} \mathrm{C}$ for $15 \mathrm{~s}$ and $60^{\circ} \mathrm{C}$ for $1 \mathrm{~min}$ for 40 cycles. Ct numbers were used to calculate the expression levels of genes of interest normalised to endogenous cellular GAPDH.

\section{Statistical analysis}

All data are expressed as mean \pm SE. Statistical analyses were performed using SPSS software version 18.0 (Chicago, IL, USA), and statistical significance was assessed through one-way analysis of variance. A value of $\mathrm{P}<0.05$ was considered statistically significant.

\section{Results}

Characterisation of hWJMSCs and their EVs

The hWJMSCs and their EVs were characterised as previously described $[22,23]$. The hWJMSC morphology was spindle-shaped and adhered to plastic surfaces. The MSC surface markers (CD44, CD73, CD90 and CD105) were positive, whereas the expressions of hematopoietic markers (CD45, CD34 and CD14) and an endothelial marker (CD31) were negative (data not shown). Transmission electron microscopy showed that EVs presented as heterogeneous lipid bilayer vesicles of approximately 30-500 $\mathrm{nm}$ in diameter and were characterised as cup-shaped or irregular-shaped (data not shown). FACS analysis showed that the EVs surface molecules were identical to their original cells (data not shown). 


\section{Kidney Blood Pressure Research}

MSC-derived EVS alleviated histological damage in AKI and saved renal function

PAS staining was performed to evaluate tubular injury levels. Tubular detachment, foamy degeneration and necrosis were observed in rat kidney sections after IRI. The most severe lesions were observed in the corticomedullary boundary area. Morphological damage in the vehicle and HFF-EV groups was much more severe than that in the MSC-EV group (Figure 1A). Tubular scores in MSC-EV group were reduced by MSC-EV treatment (Figure 1B). The renal function reflected by serum creatinine and BUN was impaired after IRI compared with that in sham-operated animals but saved by MSC-EV treatment compared with the vehicle group. By contrast, HFF-EV

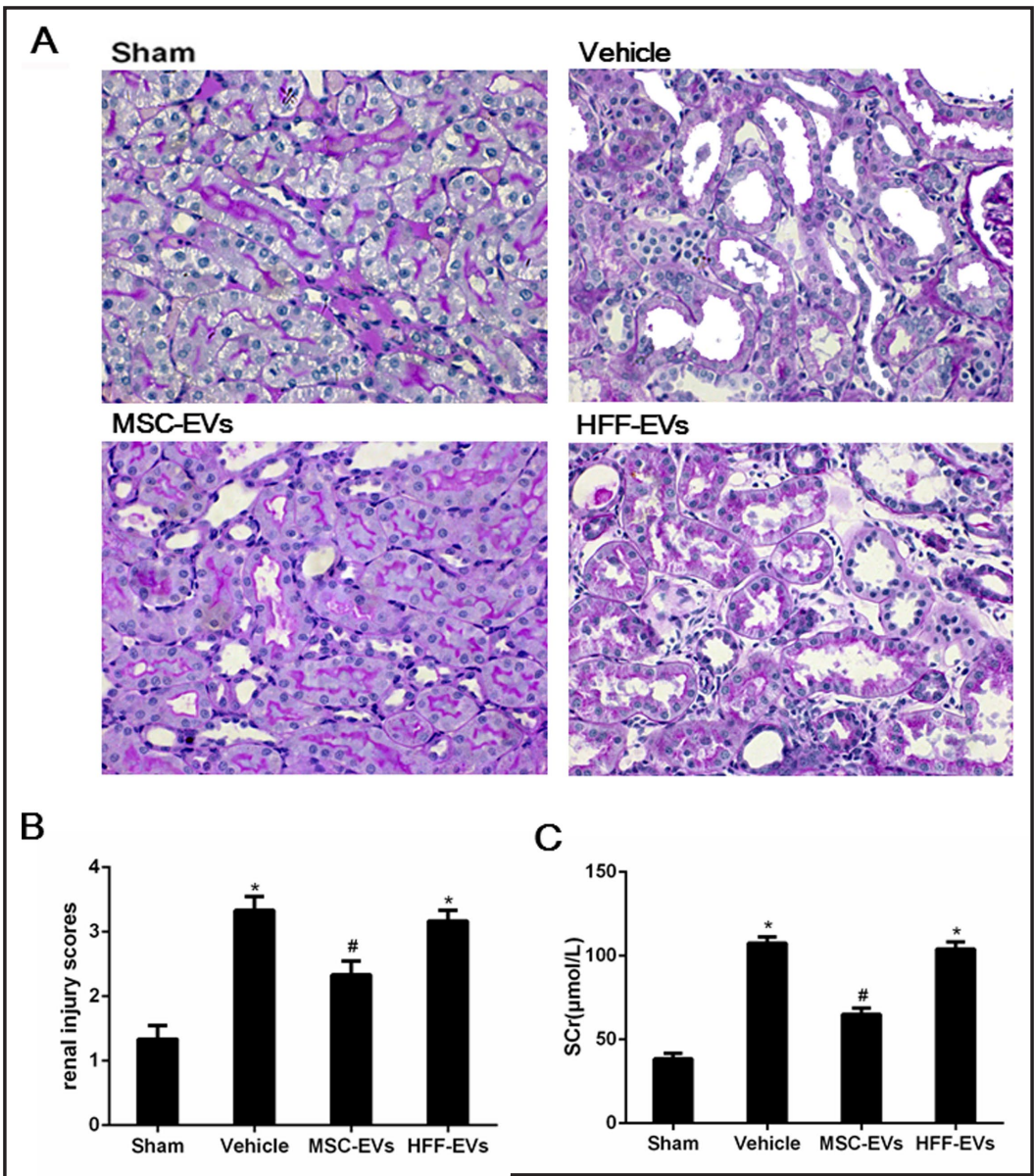

D

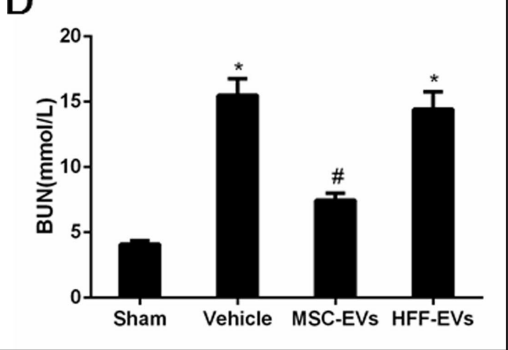

Fig. 1. MSC-EVs ameliorated renal histological damage and improved renal function. A. Representative renal sections from the sham, vehicle, MSC-EV and HFF-EV groups (PAS, 400x). B. Renal injury scoring and quantitative analysis. C, D. Serum creatinine and blood nitrogen urea from the sham, vehicle, MSC-EV and HFF-EV groups. $* \mathrm{P}<0.05$ versus the sham group. \#P $<$ 0.05 versus the vehicle group. (Figures 1C, 1D).

Renal cell injury and apoptosis were mitigated by MSC-EV administration

Serum NGAL, a classic AKI biomarker [30], was measured to evaluate kidney cell injury using ELISA. sNGAL levels were much more elevated after $24 \mathrm{~h}$ of IRI than in sham-operated animals and declined with administration of MSCs-EVs but not HFF-EVs (Figure 2C). Based on TUNEL assay of kidney tissue sections, apoptotic cell counts increased after IRI, and the number of TUNEL positive cells was reduced by MSC-EV infusion. HFF-EVs could not similarly mitigate kidney cell apoptosis (Figures 2A, 2B).

MSC-EVs enhanced Nrf2 activation, up-regulated HO-1 expression and alleviated oxidative stress in injured kidney

Elevated MDA and 8-OHdg levels in kidney tissues were observed after injury. Reduced 


\section{Kidney Blood Pressure Research}

Fig. 2. MSC-EVs reduced kidney cell injury and apoptosis. A. Representative renal sections from the sham, vehicle, MSC-EV and HFF-EV groups (TUNEL assay, 400×). B. Quantitative analysis of tubular cell apoptosis. C. Serum NGAL levels from the sham, vehicle, MSC-EV and HFF-EV groups. $* \mathrm{P}<0.05$ versus the sham group. $\# \mathrm{P}<0.05$ versus the vehicle group.

MDA and 8-OHdg levels could be seen after MSC-EV treatment, indicating that oxidative stress was alleviated (Figures 3A, 3B). By contrast, HFF-EVs could not

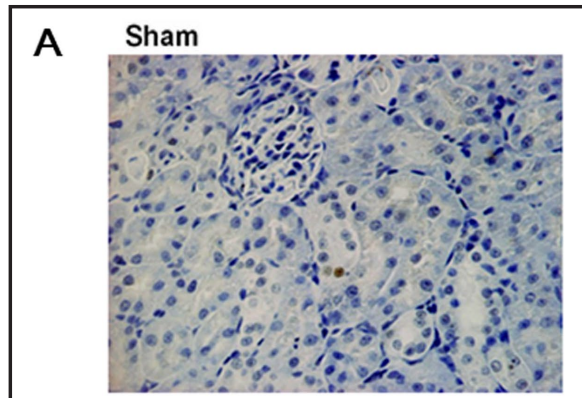
MSC-EVs
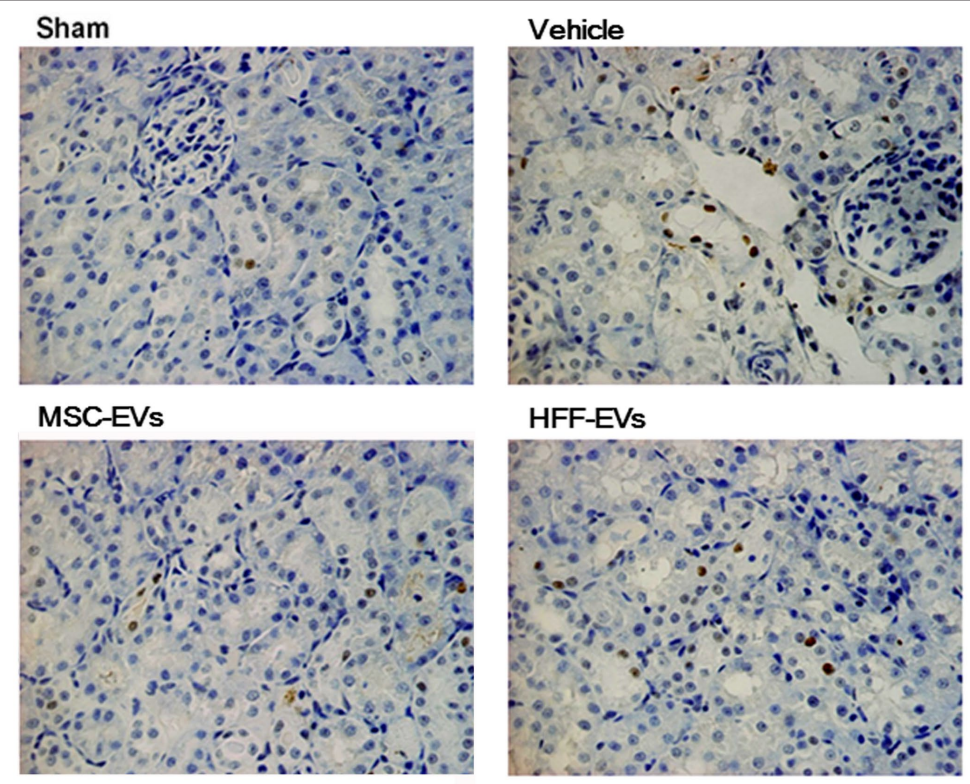

HFF-EVs

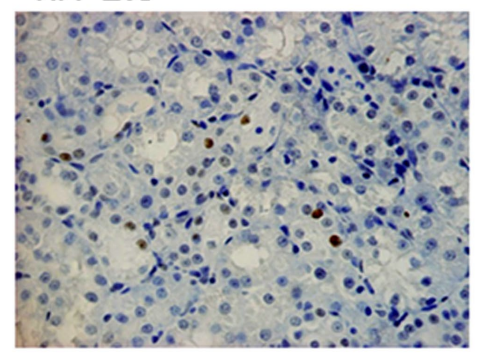

B

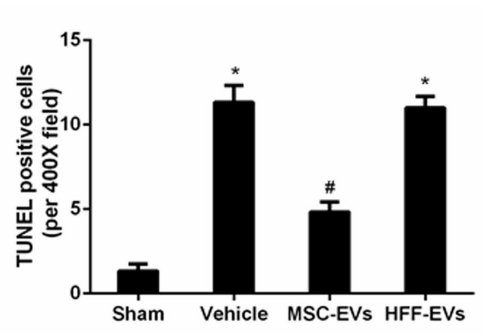

C

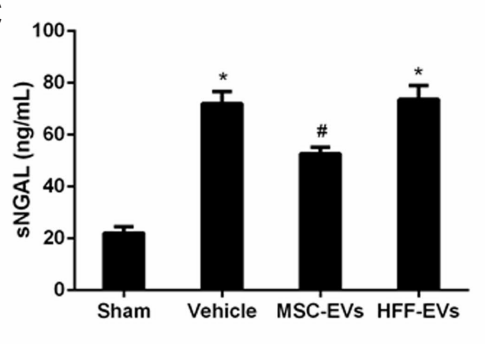
reduce MDA and 8-OHdg levels. We evaluated Nrf2 activation in the nucleus to explore the mechanism of MSC-EV anti-oxidation and found that Nrf2 was activated after IRI stress. This activation was enhanced after MSC-EV treatment (Figure 3D). Moreover, the expression of HO-1, which is the downstream gene of Nrf2, was more up-regulated in kidney tissues of the MSC-EV group than in the corresponding tissues of the vehicle group (Figure 3D). Decreased SOD levels were also observed after IRI, and MSC-EVs showed increased SOD levels in kidney tissues (Figure 3C). Similar to previous results, HFF-EVs could not enhance Nrf2 activation.

ROS level declined after MSC-EV treatment in NRK-52E cells under hypoxia injury model, and enhanced Nrf2 activation was observed.

Hypoxia re-oxygenation was performed on NRK-52E cells to confirm the influence of MSCs-EVs on Nrf2. As expected, ROS levels increased $24 \mathrm{~h}$ after hypoxia injury and MSC-EVs reduced ROS levels (Figures 4A, 4B). Nrf2 activation was also observed after injury. Moreover, enhanced activated Nrf2 (Figure 4C) and ARE activity (Figure 4D) along with up-regulated HO-1 (Figure 4E) were found in the MSC-EV group compared with those in the vehicle group. Neither enhanced Nrf2/ARE nor up-regulated HO-1 was found after HFF-EV treatment, and HFF-EVs could not decrease the ROS level.

\section{Discussion}

In this study, we observed that renal tubular injury was alleviated and renal function was improved after a single administration of MSC-EVs. Cell apoptosis and sNGAL levels, which reflect kidney cell injury, were also reduced. MSC-EVs decreased oxidative stress in 


\section{Kidney Blood Pressure Research}

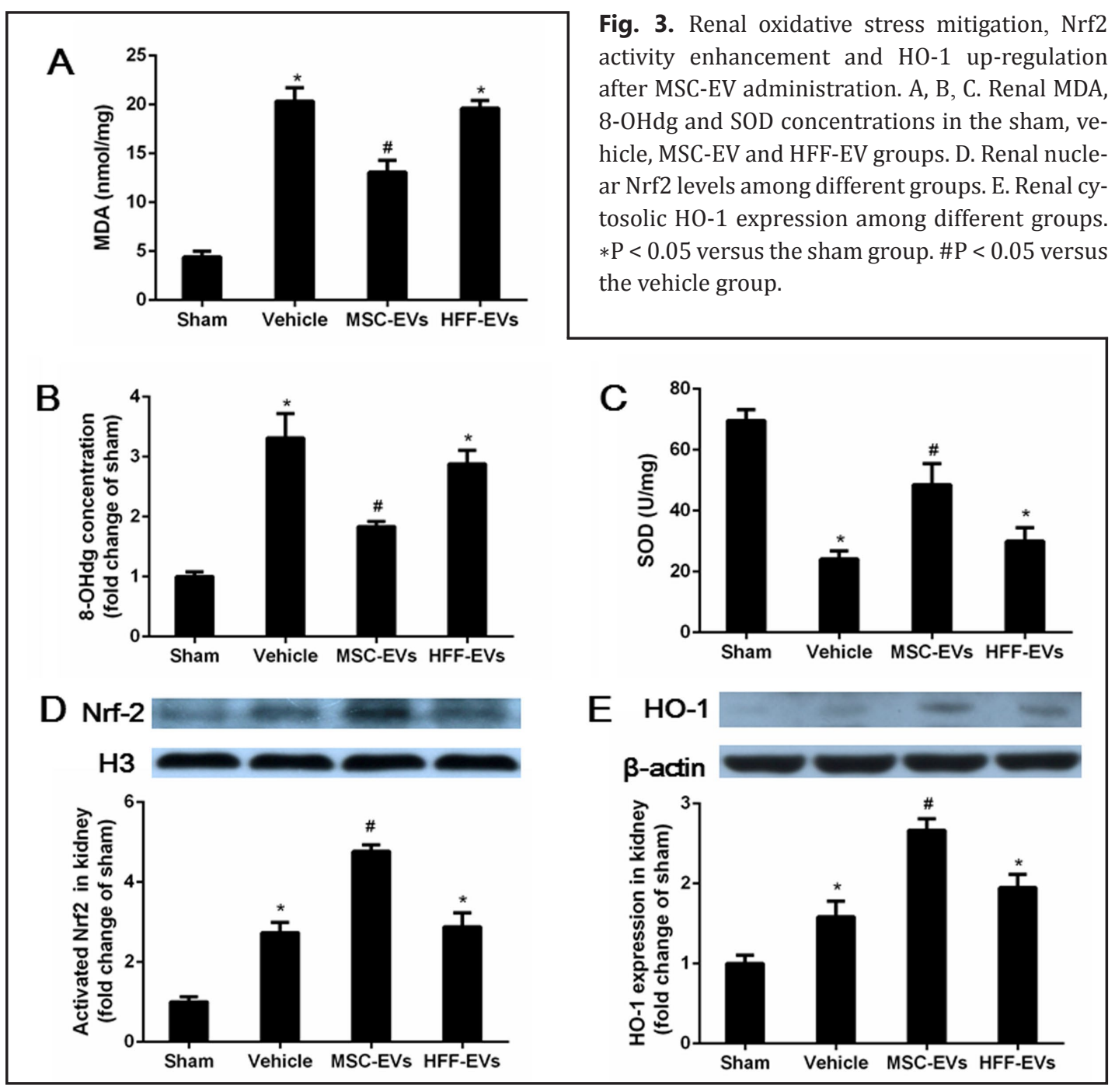

injured kidney tissues and NRK-52E cells after hypoxia injury. Nrf2/ARE enhancement and HO-1 up-regulation were observed after MSC-EV treatment both in vivo and in vitro.

MSC transplantation for tissue repair has been developed for several decades now, and favourable results have been obtained. Endocrine/paracrine mechanism rather than direct differentiation is currently considered as the main mechanism of MSC therapy [31]. 'Cellfree' MSC therapy based on the endocrine/paracrine mechanism, which takes advantage of conditioned medium or EVs of MSCs, has been suggested [7] to avoid the limitations of MSC transplantation to some extent [6]. EVs secreted by cells are membranous structures that can deliver proteins, RNA and DNA from the original cell to target cells. In this study, the EVs in CM were isolated, characterised and applied to AKI recovery in a rat model. Results indicated that EVs derived from MSCs but not HFFs can alleviate ischemia-induced AKI. Moreover, no anti-oxidative effects by HFF-EVs were observed in AKI recovery. These findings indicate that the therapeutic potential of EVs may depend on their original cells.

Previous studies have described the application of MSC-derived EV to treat various tissue injuries, including AKI, and a number of mechanisms have been proposed. The present study demonstrated that hWJMSC-derived EVs could protect against IRI by suppressing NOX2 or CX3CL1 [20, 21]. Decreased MDA and 8-OHdg levels after EV treatment were observed in the present study, indicating the anti-oxidative role of EVs in the unilateral renal IRI model.

MSCs can restore liver injury induced by $\mathrm{CCl}_{4}$ through enhancement of Nrf2 [19], thus indicating that MSC-EVs may play the same role in AKI recovery. In the present study, 


\section{Kidney Blood Pressure Research}

Fig. 4. MSC-EVs enhanced Nrf2/ ARE activation, up - regulated HO-1 expression and reduced ROS levels in NRK-52E cells under hypoxia re-oxygenation. A. Representative micrographs of DCFH-DA-labelled (green) NRK-52E cells and DAPI stained nucleus (blue) as well as merged images (DCFHDA assay, 400x). B. ROS levels in NRK-52E cells from the control, vehicle, MSC-EV and HFF-EV groups. C. Nuclear Nrf2 levels in NRK52E cells among different groups. D. ARE activity representing the transcriptional activity of $\mathrm{Nrf} 2$ was significantly increased in the MSC-EV group. E. HO-1 mRNA expression in NRK-52E cells among different groups. $* \mathrm{P}<0.05$ versus the control group. \#P < 0.05 versus the vehicle group.

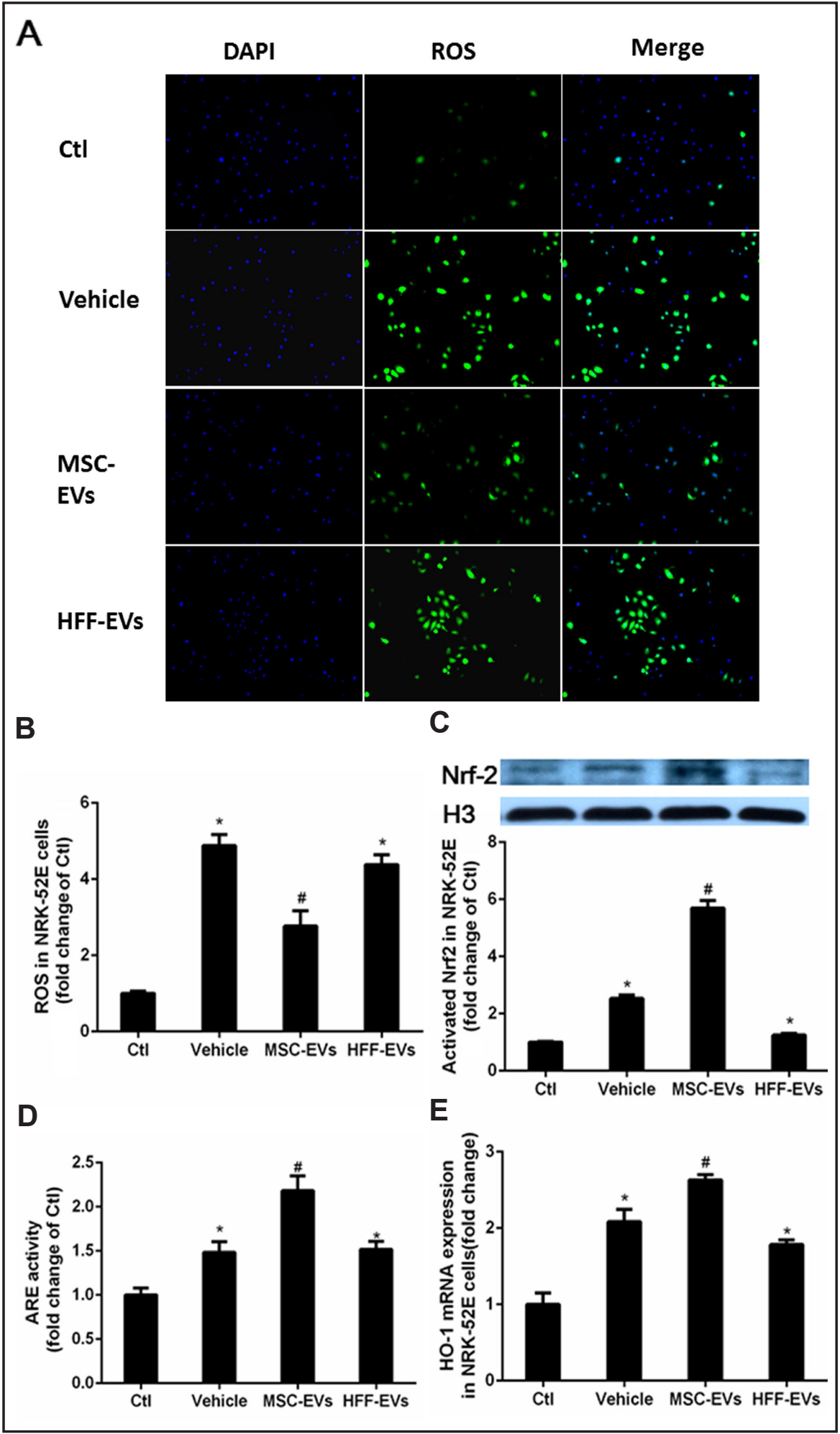

Nrf2/ARE was enhanced after EV treatment both in vivo and in vitro. HO-1 and SOD, which are ARE-regulated antioxidant enzymes, were also up-regulated by MSC-EVs. Under nonstressed conditions, Nrf2 is maintained at low levels through rapid degradation by Keap1dependent ubiquitin conjugation [32, 33]. The Nrf2 activation mechanism includes direct 


\section{Kidney \\ Blood Pressure Research}

Kidney Blood Press Res 2016;41:119-128

\begin{tabular}{l|l}
\hline DOI: 10.1159/000443413 & (c 2016 The Author(s). Published by S. Karger AG, Base \\
\hline
\end{tabular}

Published online: February 20, 2016 www.karger.com/kbr

Zhang/Zou/Huang/Wang/Miao/Liu/Chen/Zhu: MSC-EVs Protect Against AKI by Enhancing $\mathrm{Nrf2}$

sensing of oxidative stress by Keap1 and kinase pathway-dependent activation. However, the detailed mechanism behind Nrf2 enhancement by MSC-EVs remains unknown. As miRNA carried in MSC-EVs has been recognised to be the main mechanism for AKI recovery [12], Nrf2 activation may be attributed to some miRNAs delivered by MSC-EVs. This supposition, however, requires further exploration in future work.

\section{Conclusion}

MSC-EVs could recover AKI induced by IRI and help balance oxidative stress/antioxidative responses to favourable levels by enhancing Nrf2/ARE activation. The results of this study provide novel insights into the mechanism of MSC-EVs for MSC therapy.

\section{Disclosure Statement}

The authors declare that they have no competing interests and nothing to disclose.

\section{Acknowledgement}

This study is supported by grants from National Natural Science Foundation of China (81170642, 81470919, 81370849, 81300472, and 81202034), Natural Science Foundation of Jiangsu Province (BL2013032 and BK2012336), high-level talents in six industries of Jiangsu Province (2013-WSW-052), and Fundamental Research Funds for the Central Universities of China (3290005420).

\section{Reference}

1 Rosner MH, Okusa MD: Acute kidney injury associated with cardiac surgery. Clin J Am Soc Nephrol 2006;1:19-32.

2 Bonventre JV: Mechanisms of ischemic acute renal failure. Kidney Int 1993;43:1160-1160.

3 Polascik TJ, Pound CR, Meng MV, Partin AW, Fray F: Partial nephrectomy: Technique complications and pathological findings. J Urol 1995;154:1312-1318.

4 Dasta JF, Kane-Gill SL, Durtschi AJ, Pathak DS, Kellum JA: Costs and outcomes of acute kidney injury (aki) following cardiac surgery. Nephrol Dial Transplant 2008;23:1970-1974.

5 Waikar SS, Liu KD, Chertow GM: Diagnosis, epidemiology and outcomes of acute kidney injury. Clin J Am Soc Nephrol 2008;3:844-861.

6 Baglio SR, Pegtel DM, Baldini N: Mesenchymal stem cell secreted vesicles provide novel opportunities in (stem) cell-free therapy. Front Physiol 2012;3:359.

7 Rani S, Ryan AE, Griffin MD, Ritter T: Mesenchymal stem cell-derived extracellular vesicles: Toward cellfree therapeutic applications. Mol Ther 2015;23:812-823.

8 Camussi G, Deregibus MC, Bruno S, Cantaluppi V, Biancone L: Exosomes/microvesicles as a mechanism of cell-to-cell communication. Kidney Int 2010;78:838-848.

9 Simons M, Raposo G: Exosomes-vesicular carriers for intercellular communication. Curr Opin Cell Biol 2009;21:575-581.

10 Gatti S, Bruno S, Deregibus MC, Sordi A, Cantaluppi V, Tetta C, Camussi G: Microvesicles derived from human adult mesenchymal stem cells protect against ischaemia-reperfusion-induced acute and chronic kidney injury. Nephrol Dial Transplant 2011;26:1474-1483.

11 Bruno S, Grange C, Deregibus MC, Calogero RA, Saviozzi S, Collino F, Morando L, Busca A, Falda M, Bussolati B, Tetta C, Camussi G: Mesenchymal stem cell-derived microvesicles protect against acute tubular injury. J Am Soc Nephrol 2009;20:1053-1067. 


\section{Kidney \\ Blood Pressure Research}

12 Collino F, Bruno S, Incarnato D, Dettori D, Neri F, Provero P, Pomatto M, Oliviero S, Tetta C, Quesenberry PJ, Camussi G: Aki recovery induced by mesenchymal stromal cell-derived extracellular vesicles carrying micrornas. J Am Soc Nephrol 2015;26:2349-2360.

13 Vaziri ND, Dicus M, Ho ND, Boroujerdi-Rad L, Sindhu RK: Oxidative stress and dysregulation of superoxide dismutase and nadph oxidase in renal insufficiency. Kidney Int 2003;63:179-185.

14 Hybertson BM, Gao B, Bose SK, McCord JM: Oxidative stress in health and disease: The therapeutic potential of Nrf2 activation. Mol Aspects Med 2011;32:234-246.

15 Moi P, Chan K, Asunis I, Cao A, Kan YW: Isolation of nf-e2-related factor 2 (nrf2), a nf-e2-like basic leucine zipper transcriptional activator that binds to the tandem nf-e2/ap1 repeat of the beta-globin locus control region. Proc Natl Acad Sci USA 1994;91:9926-9930.

16 Wang C, Li C, Peng H, Ye Z, Zhang J, Liu X, Lou T: Activation of the nrf2-are pathway attenuates hyperglycemia-mediated injuries in mouse podocytes. Cell Physiol Biochem 2014;34:891-902.

17 Thimmulappa RK, Mai KH, Srisuma S, Kensler TW, Yamamoto M, Biswal S: Identification of nrf2-regulated genes induced by the chemopreventive agent sulforaphane by oligonucleotide microarray. Cancer Res 2002;62:5196-5203.

18 Chen YT, Sun CK, Lin YC, Chang LT, Chen YL, Tsai TH, Chung SY, Chua S, Kao YH, Yen CH, Shao PL, Chang KC, Leu S, Yip HK: Adipose-derived mesenchymal stem cell protects kidneys against ischemia-reperfusion injury through suppressing oxidative stress and inflammatory reaction. J Transl Med 2011;9:51.

19 Cho KA, Woo SY, Seoh JY, Han HS, Ryu KH: Mesenchymal stem cells restore ccl4-induced liver injury by an antioxidative process. Cell Biol Int 2012;36:1267-1274.

20 Zhang G, Zou X, Miao S, Chen J, Du T, Zhong L, Ju G, Liu G, Zhu Y: The anti-oxidative role of micro-vesicles derived from human wharton-jelly mesenchymal stromal cells through nox2/gp91(phox) suppression in alleviating renal ischemia-reperfusion injury in rats. PloS One 2014;9:e92129.

21 Zou X, Zhang G, Cheng Z, Yin D, Du T, Ju G, Miao S, Liu G, Lu M, Zhu Y: Microvesicles derived from human wharton's jelly mesenchymal stromal cells ameliorate renal ischemia-reperfusion injury in rats by suppressing cx3cl1. Stem Cell Res Ther 2014;5:40.

22 Wu S, Ju GQ, Du T, Zhu YJ, Liu GH: Microvesicles derived from human umbilical cord wharton's jelly mesenchymal stem cells attenuate bladder tumor cell growth in vitro and in vivo. PloS One 2013;8:e61366.

23 Du T, Cheng J, Zhong L, Zhao XF, Zhu J, Zhu YJ, Liu GH: The alleviation of acute and chronic kidney injury by human wharton's jelly-derived mesenchymal stromal cells triggered by ischemia-reperfusion injury via an endocrine mechanism. Cytotherapy 2012;14:1215-1227.

24 Melnikov VY, Faubel S, Siegmund B, Lucia MS, Ljubanovic D, Edelstein CL: Neutrophil-independent mechanisms of caspase-1-and il-18-mediated ischemic acute tubular necrosis in mice. J Clin Invest 2002;110:1083-1091.

25 Yang F, Huang X, Yi T, Yen Y, Moore DD, Huang W: Spontaneous development of liver tumors in the absence of the bile acid receptor farnesoid x receptor. Cancer Res 2007;67:863-867.

26 Yang R, Le G, Li A, Zheng J, Shi Y: Effect of antioxidant capacity on blood lipid metabolism and lipoprotein lipase activity of rats fed a high-fat diet. Nutrition 2006;22:1185-1191.

27 Zhu L, Yuan H, Guo C, Lu Y, Deng S, Yang Y, Wei Q, Wen L, He Z: Zearalenone induces apoptosis and necrosis in porcine granulosa cells via a caspase-3-and caspase-9-dependent mitochondrial signaling pathway. J Cell Physiol 2012;227:1814-1820.

28 Guo C, He Z, Wen L, Zhu L, Lu Y, Deng S, Yang Y, Wei Q, Yuan H: Cytoprotective effect of trolox against oxidative damage and apoptosis in the nrk-52e cells induced by melamine. Cell Biol Int 2012;36:183-188.

29 Kim ES, Lee JS, Akram M, Kim KA, Shin YJ, Yu JH, Bae ON: Protective activity of dendropanax morbifera against cisplatin-induced acute kidney injury. Kidney Blood Press Res 2015;40:1-12.

30 Bolignano D, Donato V, Coppolino G, Campo S, Buemi A, Lacquaniti A, Buemi M: Neutrophil gelatinaseassociated lipocalin (ngal) as a marker of kidney damage. Am J Kidney Dis 2008;52:595-605.

31 Bi B, Schmitt R, Israilova M, Nishio H, Cantley LG: Stromal cells protect against acute tubular injury via an endocrine effect. J Am Soc Nephrol 2007;18:2486-2496.

32 Kwak M-K, Wakabayashi N, Greenlaw JL, Yamamoto M, Kensler TW: Antioxidants enhance mammalian proteasome expression through the keap1-nrf2 signaling pathway. Mol Cell Biol 2003;23:8786-8794.

33 McMahon M, Itoh K, Yamamoto M, Hayes JD: Keap1-dependent proteasomal degradation of transcription factor nrf2 contributes to the negative regulation of antioxidant response element-driven gene expression. J Biol Chem 2003;278:21592-21600. 